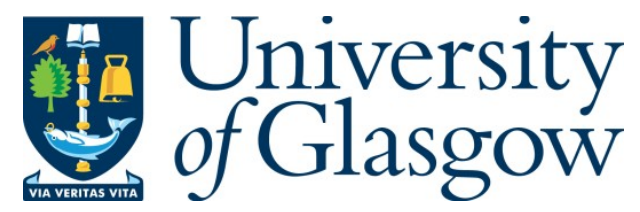

Cito, M., Kojima, O., Stevens, B. J., Mukai, T. and Hogg, R. A. (2021) Fitting of photoluminescence spectra for structural characterisation of high current density resonant tunnelling diodes for THz applications. In: SPIE OPTO, Online Only, California, United States, 6-12 March 2021.

(doi: $\underline{10.1117 / 12.2582781)}$

This is the Author Accepted Manuscript.

There may be differences between this version and the published version. You are advised to consult the publisher's version if you wish to cite from it.

http://eprints.gla.ac.uk/245639/

Deposited on: 13 July 2021

Enlighten - Research publications by members of the University of Glasgow http://eprints.gla.ac.uk 


\title{
Fitting of photoluminescence spectra for structural characterisation of high current density resonant tunnelling diodes for $\mathrm{THz}$ applications
}

\author{
Michele Cito*a, Osamu Kojima ${ }^{\mathrm{b}}$, Ben J. Stevens ${ }^{\mathrm{c}}$, Toshikazu Mukai ${ }^{\mathrm{d}}$, Richard A. Hogg ${ }^{\mathrm{a}}$

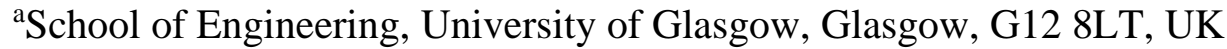 \\ ${ }^{b}$ Dept. of Electrical and Electronic Engineering, Kobe University, Kobe 657-8501, Japan \\ 'IQE plc., Cardiff, CF3 0LW, UK \\ ${ }^{\mathrm{d}}$ ROHM Co., Ltd., Ukyo-ku, Kyoto 615-8585, Japan
}

\begin{abstract}
High-resolution X-ray diffraction (HR-XRD), and low-temperature photoluminescence spectroscopy (LT-PL) are used to investigate the structural properties and inhomogeneities of high current density InGaAs/AlAs/InP resonant tunnelling diode (RTD) wafer structures. The non-destructive assessment of these structures is challenging, with structural variables: well and barriers thickness and the well indium molar fraction, in addition to electronic variables such as the band-offsets being functions of strain, growth sequence, etc.. Experimental PL data are compared with simulations allowing the deconvolution of the PL spectra, that includes Type I and Type II transitions broadened by interface fluctuations on length scales smaller and much larger than the exciton. This method provides details of the non-uniformity of the epitaxial material nondestructively.
\end{abstract}

Keywords: resonant tunneling diodes, photoluminescence, epitaxy

\section{INTRODUCTION}

The societal need for fast, ubiquitous wireless technologies has increased in the last decade[1], with the covid-19 pandemic amplifying this need due to the increase of remote working and in the online entertainment demand. To satisfy the demand of high-frequency devices researchers are investigating wireless sources (both electrical and optical) able to operate in the Tera Hertz spectrum (0.1-10 THz)[2]. From the electronic side, resonant tunneling diode (RTD) based oscillators have recently reached a maximum oscillation frequency of $1.98 \mathrm{THz}$ demonstrating that RTD technology can achieved the required frequency ranges for application. Wireless data transmission at 1.5 Gbit/s using an RTD-based oscillator was demonstrated by Mukai et. al [3].

We previously demonstrated how the RTD valley current and the device performance are not only limited by thermal effects, but mainly by interface scattering [4], with only $28 \%$ of the valley current being thermally activated, leaving space for significant improvement of the crystal quality. To optimize the epitaxial process, we first demonstrated the use of PL as rapid non-destructive characterization process[5]. Further work demonstrated that the combination of PL, HRXRD and the inclusion of a buried undoped copy QW allowed a complete structural characterization scheme for the RTD wafer [6]. Comparison with TEM underlined how critical structural parameter can be derived from this nondestructive post-growth characterization scheme [7].

In this paper we describe PL spectra deconvolution to obtain information regarding the spatial uniformity of the epitaxial wafer. First, variations from the designed structure, introduced by the growth process are presented, with a description of their impact on the characterization process. Low-temperature PL data is compared with simulation, and a set of rules are described and applied to obtain a rigorous fit to this PL spectrum incorporation long and short-range disorder of the quantum well. 


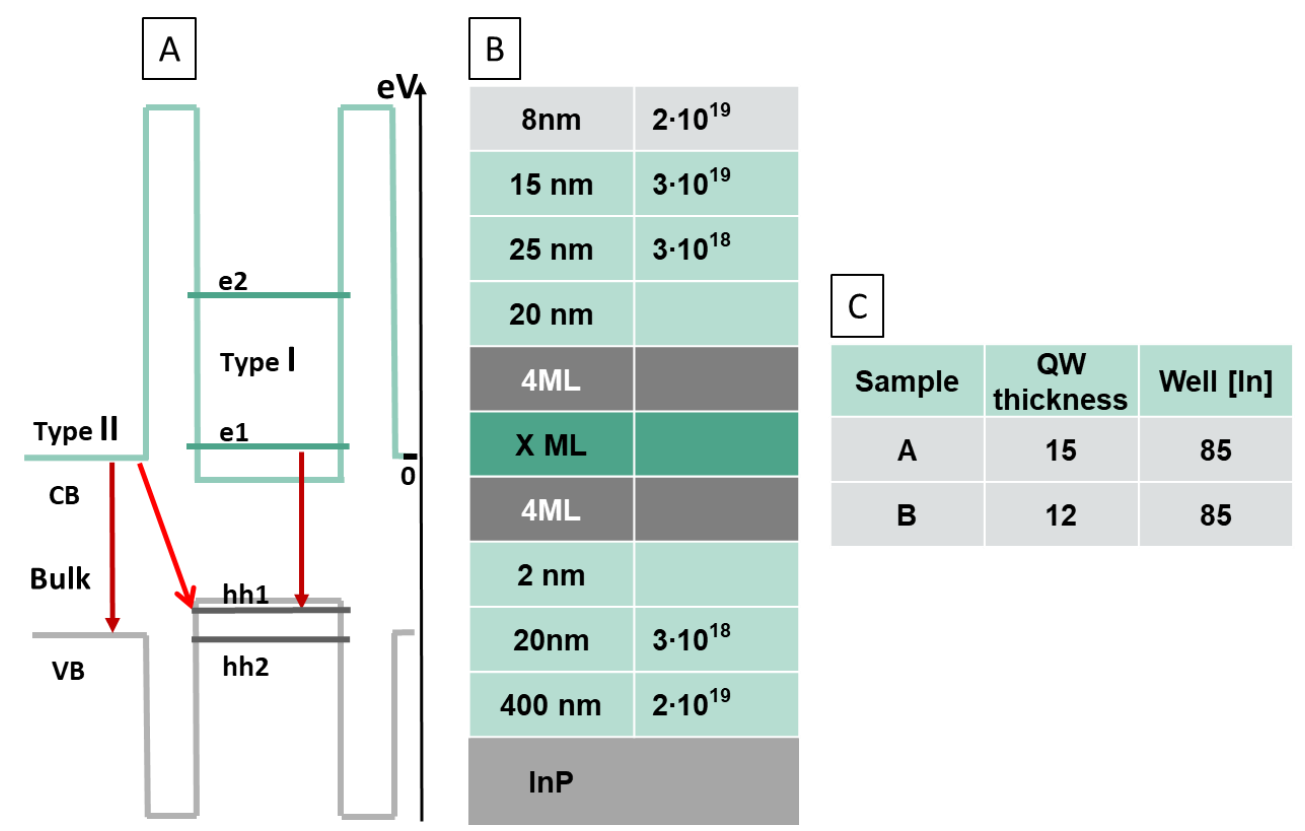

Figure 1 Schematic $Q W$ band profile (A) with the first two quasi-bound states(horizontal lines) and optical transitions (Red-lines).

RTD complete epi-structure with doped emitter and collector and contact cap (B), focus on the $Q W$ characteristic of the samples studied in this paper reported in $(C)$

Fig1 shows a schematic representation of the conduction and valence band profile, with the quasi-bound states indicated by horizontal lines. For simplicity, this schematic does not include the effect of the doping and resultant band bending (although this is taken into account in our calculations). Solid red lines highlight the optical transitions observable by PL. The Type I transition only involves the quasi bound states from e1 to hh1. The Type II transition originate from electrons in the bulk InGaAs conduction band to hh1 in the quantum well. While the bulk transition goes from the InGaAs conduction band to the valence band. The vertical black axis indicates the energy with the origin set at the bulk conduction band as reference. Figure1(B) indicates the designed epitaxial structure of the samples analysed here, with their QW characteristics reported in figure1(C). The structures have been realized to investigate the intrinsic resonator efficiency[8] varying the well thickness, to be reported elsewhere.

The structures were grown in a vertical Thomas Swan close-coupled showerhead MOVPE reactor on (100) semiinsulating InP:Fe substrates. Further details about the growth process are reported elsewhere [6]. The epitaxy starts with $100 \mathrm{~nm}$ InP buffer layer followed by $25 \mathrm{~nm}$ and $200 \mathrm{~nm}$ In0.53Ga0.47As. $400 \mathrm{~nm}$ highly n-doped In0.53Ga0.47As $(2 \times 1019 \mathrm{~cm}-3 \mathrm{Si})$ are grown for the lower contact. A $20 \mathrm{~nm}$-doped $\mathrm{In} 0.53 \mathrm{Ga} 0.47 \mathrm{As}(3 \times 1018 \mathrm{~cm}-3 \mathrm{Si})$ emitter layer is then grown, followed by a $2 \mathrm{~nm}$ In0.53Ga0.47As spacer layer. An InGaAs quantum well is formed between two $1.1 \mathrm{~nm}$ AlAs barriers. In high Jpeak design, like the ones described here, the well thickness is always lower than $4.4 \mathrm{~nm}$ and

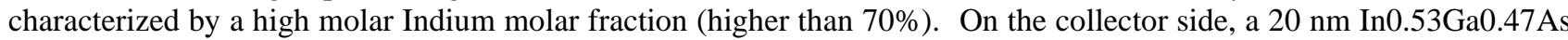
spacer layer is grown with a $25 \mathrm{~nm}$ In0.53Ga0.47As $(3 \times 1018 \mathrm{~cm}-3 \mathrm{Si})$ collector layer. The epitaxy is terminated with 15

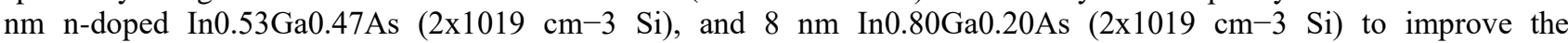
conductivity at the collector side.

\section{PHOTOLUMINESCENCE SPECTROSCOPY}

Low temperature PL was performed in a closed cycle helium cryostat, with the sample at a temperature of $4 \mathrm{~K}$. A tunable Ti:sapphire laser was used to excite the sample with a power density of $\sim 1 \mathrm{~W} / \mathrm{cm}^{2}$. The PL signal was dispersed by a Sol Solar T II double monochromator and detected by an InGaAs transimpedance amplified photodetector. A resolution of 
$5 \mathrm{~nm}$ was used. The modelling package[9] first determines the band profile of the entire structure at $4 \mathrm{~K}$, including both strain and the asymmetric doping of the device. Band line-ups are computed using the model-solid theory[10]. Schroedinger-Poisson equations were solved self-consistently indicating the extension of the QW region.
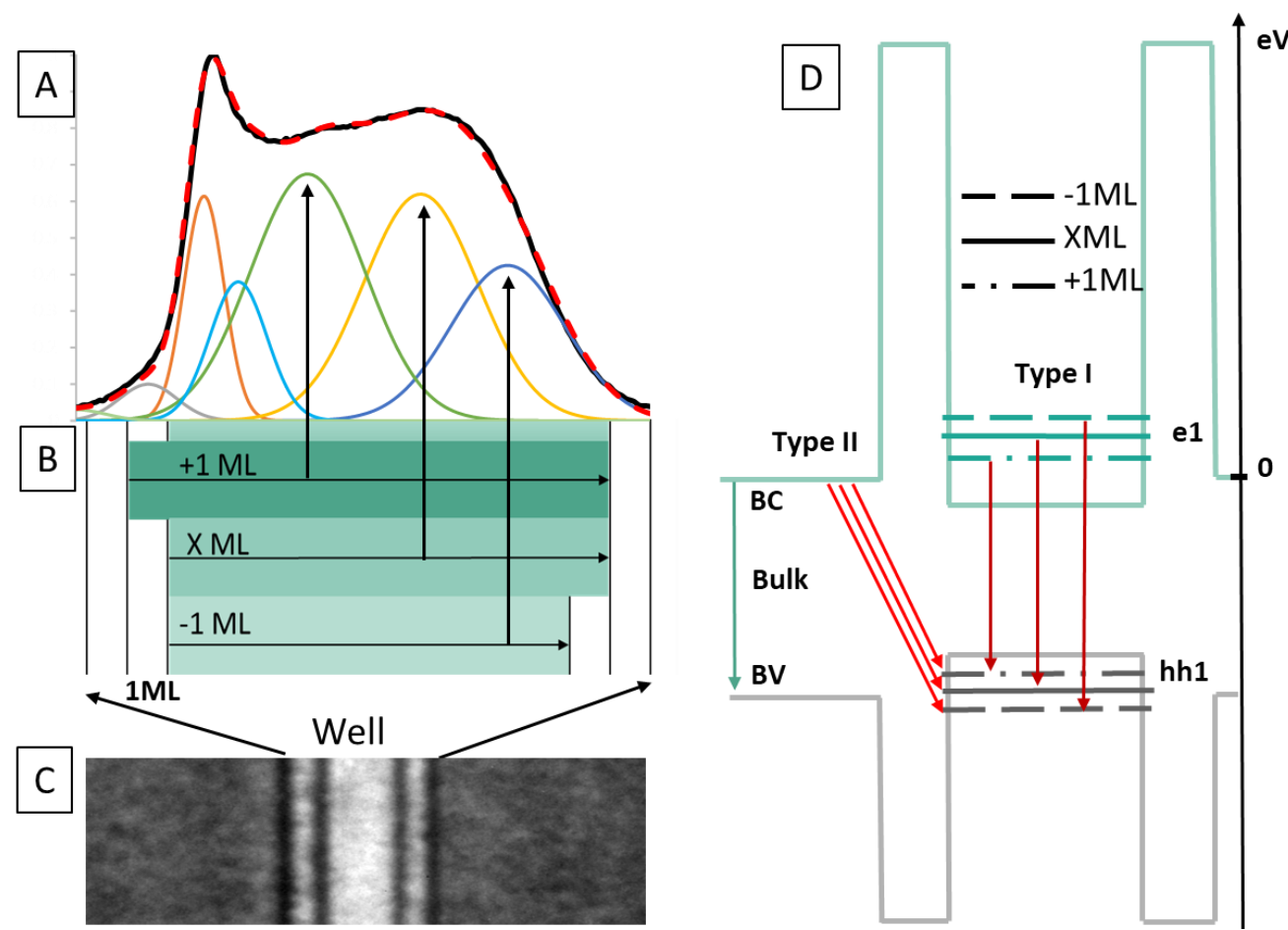

Figure 2Schematic illustrating effect of monolayer fluctuation starting from a TEM scan micrograph(C) to the impact on the PL characterization (B and A). Schematic band profile including the additional el-hhl states and optical transition generated by the $M L$ fluctuation.

The group of schematics and micrographs reported in figure2 are intended to illustrate the mono-layer fluctuations present withing our samples at the heterointerfaces and their impact on the PL characterization. Starting from the bottom, figure 2(C) shows a representative darkfield TEM image of the RTD active region, in which it is possible to observe the two barriers and the well region. Figure 2(B) shows a schematic representation of the ML-fluctuation and their impact upon the confinement energy within the QW, and associated optical transitions, shown in figure 2(D). Figure 1(A) finally shows typical low temperature PL spectra: the barrier roughness (length scale < exciton diameter) creates broad PL peaks while the ML-fluctuation (length scale > exciton diameter) cause peak splitting and an overall broadening of the PL trace [11]. The origin of the peak splitting is clarified numerically in fiure2(D): the ML-fluctuation creates 2 additional quasi-bound states $( \pm 1 \mathrm{ML})$ in addition to the designed one (XML). The Type I transition is now a triplet, and the same happens for the Type II transition[6]. Careful analysis of the Type I and Type II peaks is crucial to measure the position of the first quasi bound state, $\mathrm{e}_{1}$, as it is given by the T1 and T2 difference [12]. In the following, we describe the deconvolution process. 

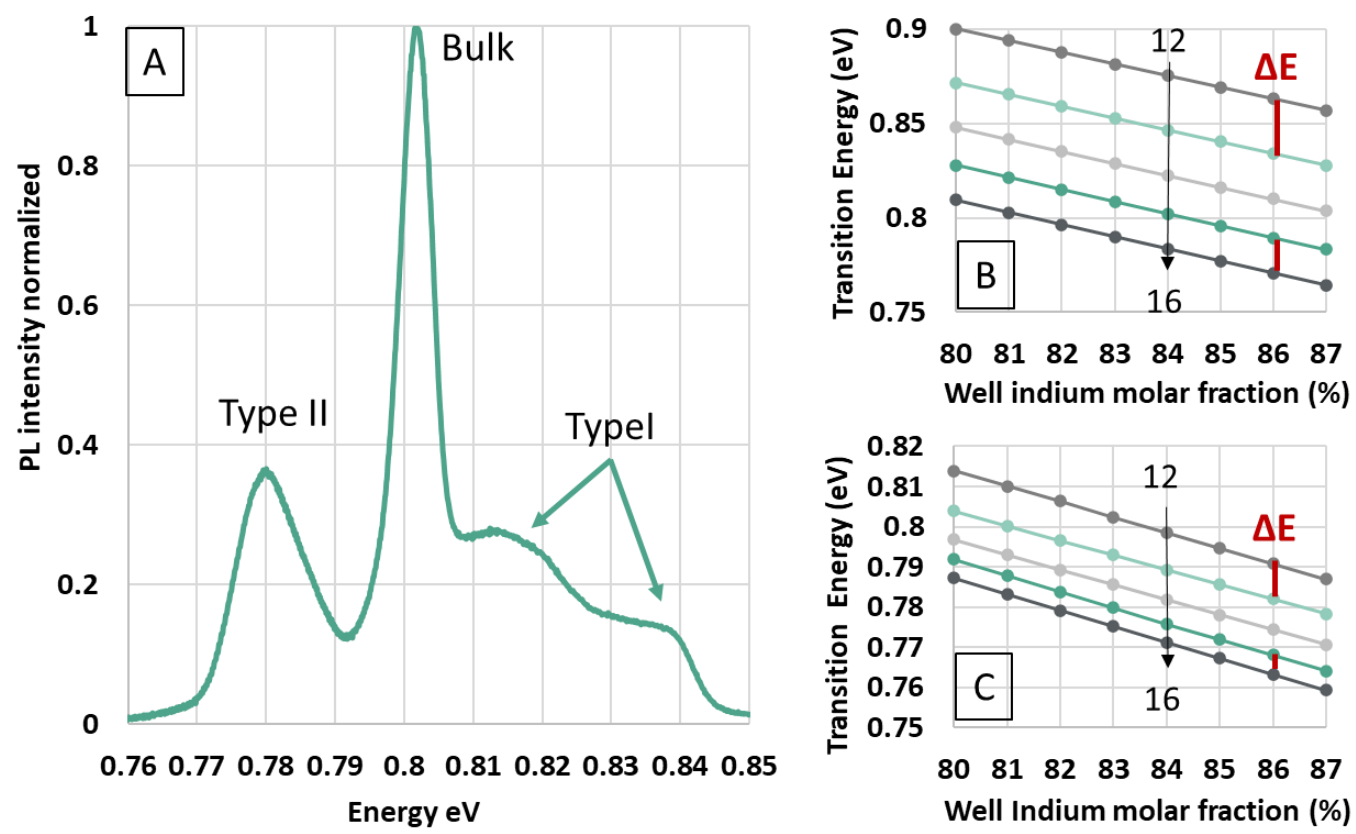

Figure 3 Experimental PL spectra (A) and simulated optical transition as function of varying the indium molar fraction and the well thickness, for Type $I(B)$ and Type II $(C)$, respectively. Red lines are highlighting the energy split between adjacent well thickness solution.

Figure 3(A) shows the 4K PL spectra measured from sample A obtained with $1 \mathrm{~mW} / \mathrm{cm}^{2}$ excitation at $900 \mathrm{~nm}(1.37 \mathrm{eV})$. Four peaks are visible to the eye, starting from lower energy we observe a broad peak at $0.78 \mathrm{eV}$ attributed at the Type II transition, the central sharp peak $(0.801 \mathrm{eV})$ attributed at the LM-InGaAs bulk peak, and two broad peaks $(0.812 \mathrm{eV}$ and $0.838 \mathrm{eV}$ ) associated with the Type I transition. As described in the introduction, a triplet from both the Type I and Type II transition are expected. For the Type I transition we can observe two of the three peaks (X and -1ML), while the expected peak at lower energy (+1ML solution) is concealed between the Type II transition and the bulk peak. Deconvolution of the spectrum is therefore required to determine the position of each radiative transitions, through a step-by-step process comparing experimental data with simulations. Figures3(B-C) plot the Type I and II transition energies obtained by simulation varying the InGaAs well indium molar fraction and the well thickness from 12MLs to $16 \mathrm{ML}$ in ML steps using a conduction band offset of $65 \%$. Red lines highlight $\Delta \mathrm{E}$ between adjacent solutions, and it is observed that this is almost constant for fluctuation of $1 \mathrm{ML}$.

We go on to explore the effect of conduction band offset on these transitions. Figure 4(A) shows the calculated transition energies as a function of the well thickness for an arbitrary indium concentration (84\%) with a standard conduction band offset (65\%), accordingly with the model-solid theory [10]. Well thickness is expressed in nm, with mono-layers solutions marked by the dots while red arrows highlight the $\Delta \mathrm{E}$ for adjacent well thicknesses. Figure4(B) shows the same simulation increasing the band offset to (75\%), the change in $\Delta \mathrm{E}$ respect figure4(A) is $0.3 \mathrm{meV}$, and is too small to be observed in the PL spectra. Based on this result we can assume that a change in band offset will not confound the line shape fitting, and the $\Delta \mathrm{E}$ will be fundamental in assigning peak position to the fit as it places a limit on possible well-widths $(-1, \mathrm{X},+1 \mathrm{MLs})$ for the structure. 

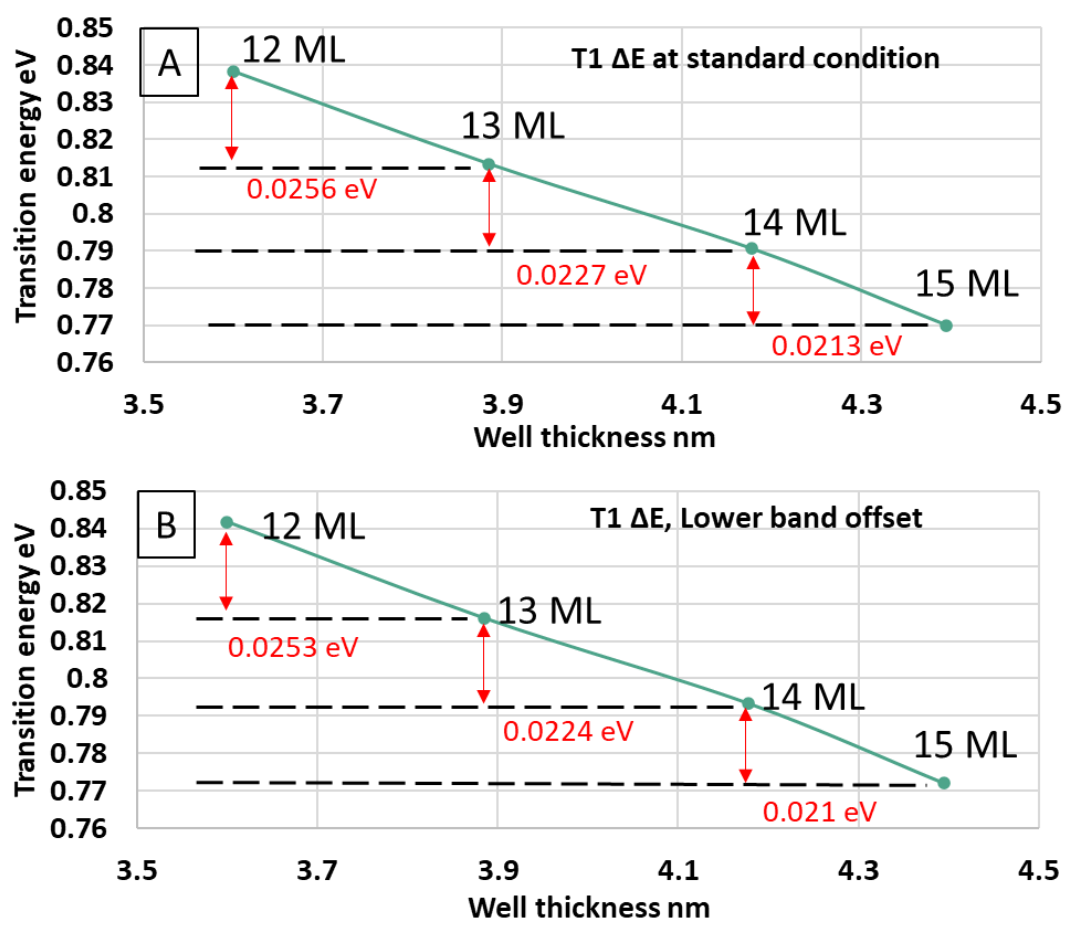

Figure 4 (A)Type I simulation at standard conduction band offset (65\%).

$(B)$ the same simulation increasing the conduction band offset (75\%). Red arrows and number are highlighting the energy split between adjacent wel thickness solution. Simulations were computed assuming an indium molar fraction of $84 \%$.

\section{LINE-SHAPE FITTING}

The line shape fitting is based on several constraints to regulate the position, shape, and the amplitude of the peaks. The deconvolution of the Type I emission is obtained using 3 Gaussian peaks $(\mu, \sigma T 1)$ (X ML, $\pm 1 \mathrm{ML}$ ). Their position on the energy axis $(\mu)$ is based on the simulation described in Figure3(B and C). Based upon the essentially equal splitting of the transitions we assume an equal linewidth $(\sigma \mathrm{T} 1)$ for the three Type I transitions[6]. For the Type II transitions, we use the same rules, with 3 Gaussian peaks $(\mu, \sigma \mathrm{T} 2)$ for the transitions (X ML, $\pm 1 \mathrm{ML}$ ). Here, the energetic position is again dictated by simulation but with the linewidths $(\sigma \mathrm{T} 2)$ governed by the variation in the hole transition energies[13]. In the deconvolution process, the ratio of the integrated intensity of the X, $+1 \mathrm{ML},-1 \mathrm{ML}$ transitions for the Type I and Type II transitions are kept constant. 


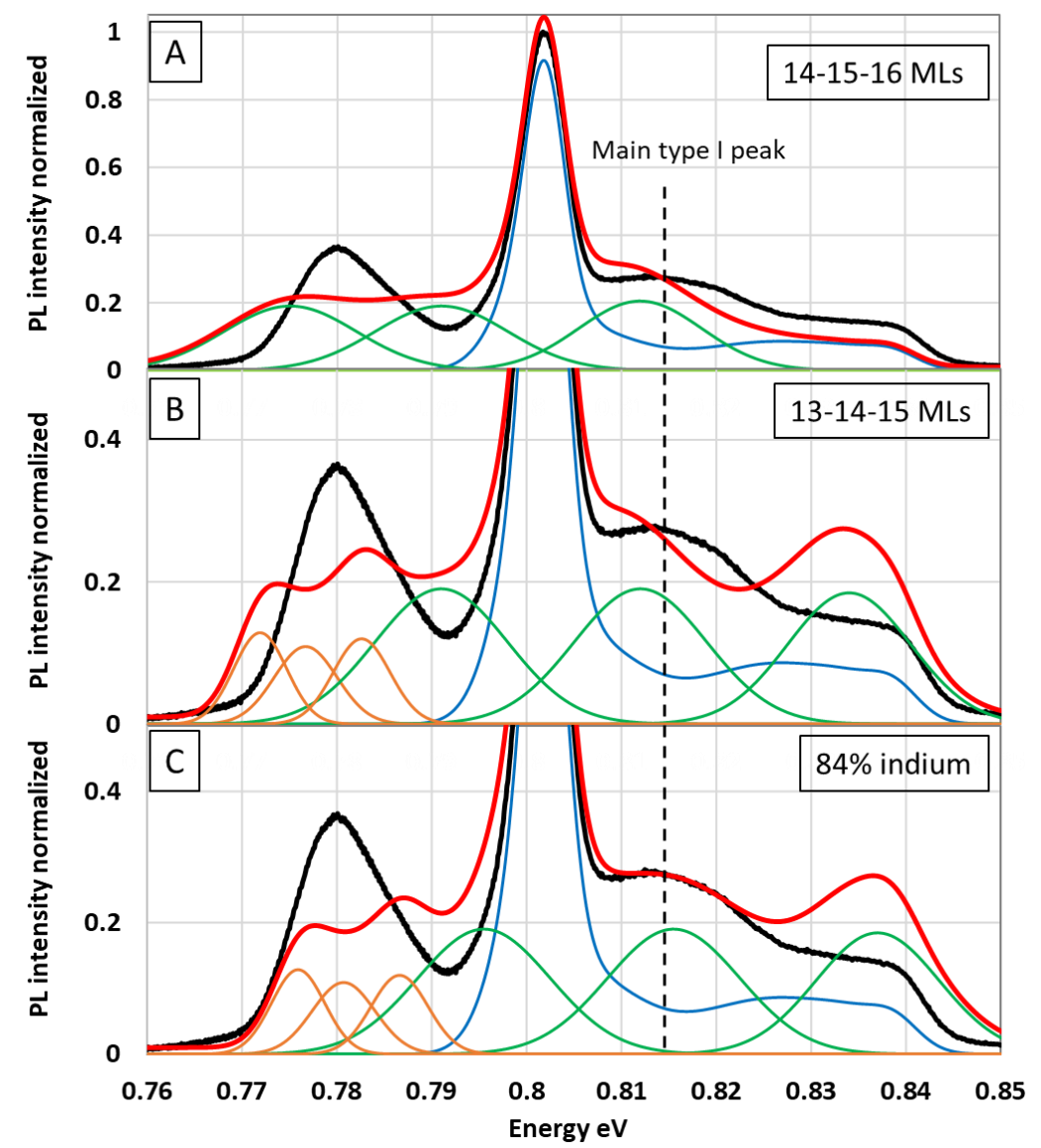

Figure 5Line shape fitting steps, (A) bulk and doped-bulk peak fitting,(B)Type I and Type II triplets matching, (C) molar fraction correction to match peaks energy. Peak intensity are arbitrary at this stage.

Figures5 illustrates schematically possible fitting steps for sample A in figure1(C). Our procedure begins by accommodating the bulk peak and the doped bulk InGaAs tail (blue curve in figure5(A)), using PL characterization of doping layers extensively studied by K.J Jacobs [14]. Based on the simulation reported in figure3(B), we then accommodate the expected 3 Type I peaks (15ML and the $\pm 1 \mathrm{ML}$ ) as shown in figure5(A). The peak amplitude is arbitrary at the stage, to simply visualize their position we settled on the same intensity values for all. We note here that in this case, the $+1 \mathrm{ML}$ peak at $0.775 \mathrm{eV}$ is partially outside of the spectra, the $\mathrm{XML}$ peak at $0.791 \mathrm{eV}$ may be fitted tuning the amplitude while the -1ML peak seems not in line with what we observe with the peak at $0.815 \mathrm{eV}$ (black dashed line). We find that no good fit can be obtained for the triplet 14-15-16 MLs. Figure 5(B) shows a possible fit using the triplet 13-14-15 MLs. Here we again obtain peaks at $\sim 0.79$ and $0.81 \mathrm{eV}$ ( +1ML and XML peak this time) due, along with a new peak at $0.834 \mathrm{eV}$ that now lies inside the envelope of the experimentally obtained curve, providing a candidate for fitting the spectrum. We are now able to accommodate the 3 Type II peaks, albeit with the +1ML feature being partially outside the envelope of the spectrum. We note that a fit may be possible by reducing its amplitude, but the major concern is the mismatch of the XML peak, that suggests that by tuning the molar fraction we may increase the transition energy and improve the fitting. Figure 5(C) shows the triplets changing the molar fraction from $85 \%$ to $84 \%$, all the peaks are inside the experimental spectra and the XML also match at $0.815 \mathrm{eV}$. Once the position of the peaks has been established, their amplitudes need to be tuned, and as we previously indicated, the ratio of Type I to Type II emission (T1/T2) is a fit parameter. This parameter occurs as we consider that the laser energy may excite differently the Type I and II transition for a given ML solution, but all the triplets from spatially separate regions of the sample are equally excited. 

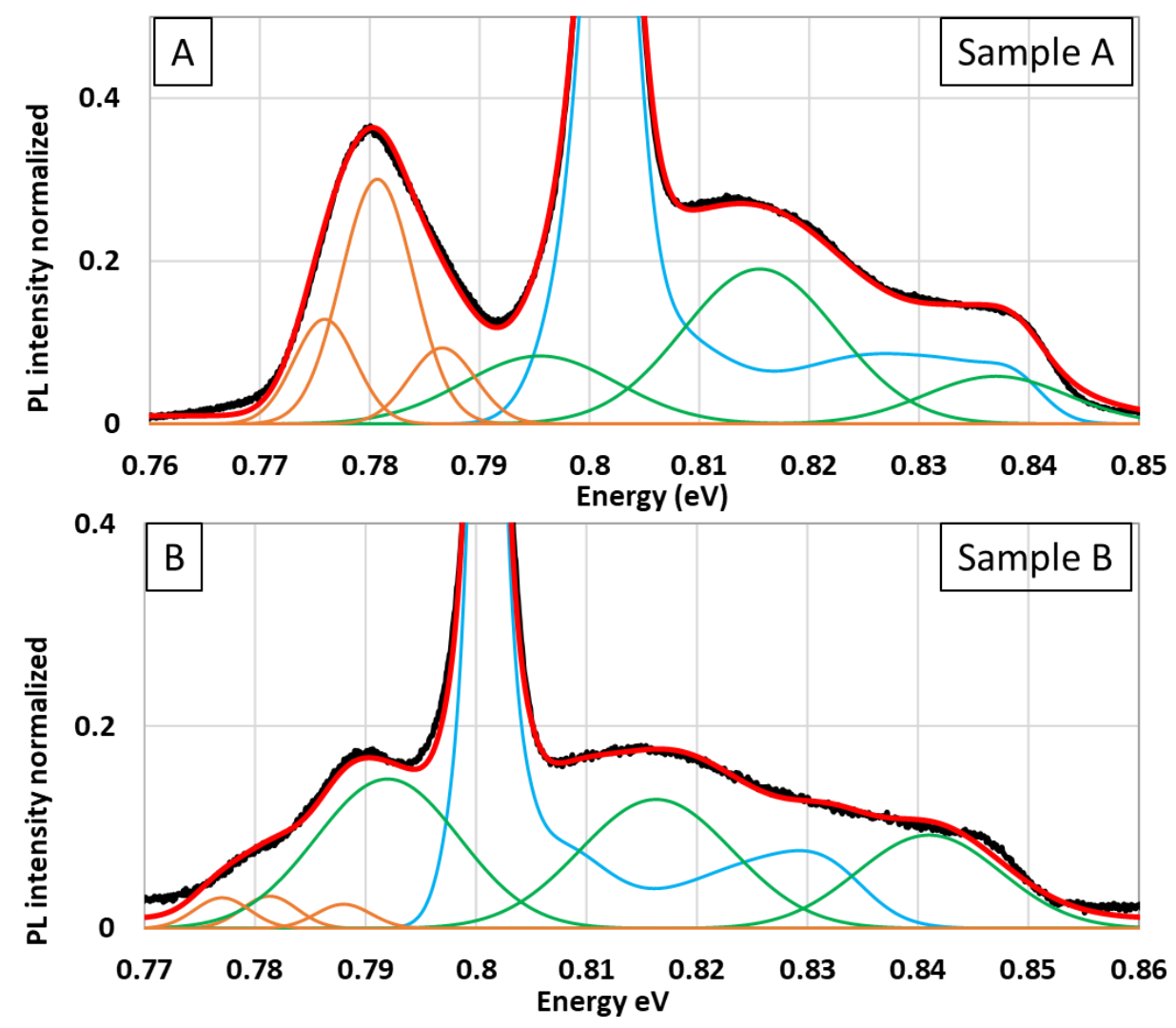

Figure 6 (A)Line shape fitting for sample A, 14MLs well 84\% [In]. (B) fitting for sample B, 12MLs well $85 \%$.

Having described the fitting process, we now move to fit the PL spectra of two different samples, combining simulation and weighting of the different peasks due to long range disorder. Figure 6(A) shows the fit obtained tuning the T1/T2 ratio with minor correction on the positions of the peaks given by variation of the band offset. The bulk peak has a linewidth of $4 \mathrm{meV}$, in agreement with the limit of $3.3 \mathrm{meV}$ predicted for perfect LM-InGaAs [11].For the fitting, we used $\sigma \mathrm{T} 1=7 \mathrm{meV}$ and $\sigma \mathrm{T} 2=3 \mathrm{meV}$ and a $\mathrm{T} 2 / \mathrm{T} 1=1.57 \pm 0.01$ utilising a band-offsets of $59 \%$. The $\mathrm{T} 1 / \mathrm{T} 2$ ratio is the additional task to validate the positions of the peak once the PL line shape is fitted. An arbitrary assignment of the amplitudes of each peak may lead to an ambiguous combination of QW thickness, indium concentration, and band offset. The XML Type II transition is at $0.781 \mathrm{eV}, 1 \mathrm{meV}$ higher with respect to the observed position without the line shape fitting. Similar considerations are made for the X ML Type I transition peak, with the e1 position being $0.0345 \mathrm{eV}$ lower with respect to our first estimation $(0.045-0.05 \mathrm{eV})$ based upon standard band offsets. Figure6(B) shows the fitting obtained for the second sample in figure1(C), in this case, the T1/T2 is $3.84 \pm 0.01$. For this sample $\Delta \mathrm{E}$ is wider, and peaks are more spread on the energy axis. For the fitting, we used $\sigma \mathrm{T} 1=6.5 \mathrm{meV}$ and $\sigma \mathrm{T} 2=3 \mathrm{meV}$, with the same band offset.

\section{CONCLUSION}

In conclusion, we have described a method to fit the PL line-shape for high Jpeak RTD structures, and how this deconvolution increases the accuracy of structural characterization. The method compares simulation and experimental data to provide a set of "fitting constraints" for an unambiguous fitting. Two different samples have been analysed to highlight the versatility of the method in case of wide peak splitting and low Type II intensity. A successful deconvolution of the PL spectra may unlock the use of PL excitation spectroscopy to assess the QW conduction band- 
offset and improve the characterization accuracy both in terms of structural and electronic paramters such as the bandoffsset [15].Furthermore, reduction of the PL spot size may lead to a better evaluation of crystal/device imperfection on length scales higher and lower than the exciton size.

Acknowledgments

This project has received funding from the European Union's Horizon 2020 research and innovation programme under the Marie Skłodowska-Curie under Grant Agreement No. 765426 (TeraApps).

[1] Cherry, S., Edholm's law of bandwidth. IEEE spectrum, 2004. 41(7): p. 58-60.

[2] Nagatsuma, T., Terahertz technologies: present and future. IEICE Electronics Express, 2011. 8(14): p. 11271142 .

[3] Shiode, T., et al. Giga-bit wireless communication at $300 \mathrm{GHz}$ using resonant tunneling diode detector. in AsiaPacific Microwave Conference 2011. 2011. IEEE.

[4] Jacobs, K., et al., Valley current characterization of high current density resonant tunnelling diodes for terahertz-wave applications. AIP Advances, 2017. 7(10): p. 105316.

[5] Jacobs, K.J., B.J. Stevens, and R.A. Hogg, Photoluminescence Characterisation of High Current Density Resonant Tunnelling Diodes for Terahertz Applications. IEICE Transactions on Electronics, 2016. 99(2): p. 181-188.

[6] Baba, R., et al., Non-destructive characterization of thin layer resonant tunneling diodes. Journal of Applied Physics, 2019. 126(12): p. 124304.

[7] Baba, R., et al. Characterisation of thin-layer resonant tunnelling diodes grown by MOVPE. in Quantum Dots and Nanostructures: Growth, Characterization, and Modeling XVI. 2019. International Society for Optics and Photonics.

[8] Baba, R., et al., Epitaxial Designs for Maximizing Efficiency in Resonant Tunneling Diode Based Terahertz Emitters. IEEE Journal of Quantum Electronics, 2018. 54(2): p. 1-11.

[9] Birner, S., et al., Nextnano: general purpose 3-D simulations. IEEE Transactions on Electron Devices, 2007. 54(9): p. 2137-2142.

[10] Van de Walle, C.G., Band lineups and deformation potentials in the model-solid theory. Physical review B, 1989. 39(3): p. 1871.

[11] Herman, M., D. Bimberg, and J. Christen, Heterointerfaces in quantum wells and epitaxial growth processes: Evaluation by luminescence techniques. Journal of Applied Physics, 1991. 70(2): p. R1-R52.

[12] Jacobs, K.J., et al. Characterisation of high current density resonant tunnelling diodes for THz emission using photoluminescence spectroscopy. in 201641 st International Conference on Infrared, Millimeter, and Terahertz waves (IRMMW-THz). 2016. IEEE.

[13] Ledentsov, N., et al., Radiative states in type-II GaSb/GaAs quantum wells. Physical Review B, 1995. 52(19): p. 14058 .

[14] Jacobs, K., et al., Non-destructive mapping of doping and structural composition of MOVPE-grown high current density resonant tunnelling diodes through photoluminescence spectroscopy. Journal of Crystal Growth, 2015. 418: p. 102-110.

[15] Duggan, G., A critical review of heterojunction band offsets. Journal of Vacuum Science \& Technology B: Microelectronics Processing and Phenomena, 1985. 3(4): p. 1224-1230. 
